\title{
Effective Cluster based Routing Algorithm for WMNS using Effective Data Aggregation
}

\author{
Preet Pavneet Kaur Sandhu \\ MTECH Student \\ Baba Banda Singh Bahadur Engineering College \\ Fatehgarh Sahib, Punjab
}

\author{
Jatinder Singh Saini \\ Asst. Prof. \\ Baba Banda Singh Bahadur Engineering College \\ Fatehgarh Sahib, Punjab
}

\begin{abstract}
Wireless Mesh Network (WMN) is a key technology, supporting a variety of several emerging and commercially interesting applications. The multi-hop nature of WMNs and the rapid growth of throughput demands lead to multichannels and multi-radios structures in mesh networks, but the interference of co-channels, as a main problem reduces the total throughput, especially in multi-hop networks. QoS (Quality of Service) refers to a vast collection of networking technologies and techniques that guarantees the ability of a network to provide with predictable results. Due to interference among various transmissions, the QoS routing in multi-hop wireless networks is formidable task. In case of multi-channel wireless network, since two transmissions using the same channel may get in with each other so there exists interference. The request of QoS connection usually accompanies bandwidth requirement and QoS routing seeks a source to destination route with requested bandwidth. The main objectives of QoS based routing are optimal utilization of resources for improving total network throughput and graceful performance degradation during overloading conditions offering better throughput, Dynamic determination of feasible paths for accommodating the QoS of the given flow under various policy constraints such as provider selection, path cost etc. The overall objective of this paper is to propose a new multi-radio, multi-channel and clustering based wireless mesh protocol to improve the QoS further. This paper has considered the DSDV protocol to locate the secure and optimized path. The proposed technique also utilizes the LZW based lossless data compression and intra cluster data aggregation to enhance the communication between the source and the destination. The use of clustering has the ability to aggregates the multiple packets and locate a single route using the clusters to improves the intra cluster data aggregation. The use of the LZW based loss less data compression has ability to reduce the data packet size so will consume lesser energy thus increase the network QoS. The MATLAB tool has been used to evaluate the effectiveness of the proposed technique. the comparative analysis has shown that the proposed technique outperforms over the available techniques.
\end{abstract}

Keywords: WMNs, QoS, DSDV, AODV, QoS IN WMNS, DATA AGGREGATION.

\section{INTRODUCTION}

Wireless Mesh Networks (WMNs) is a key technology, supporting a variety of several applications._Wireless Mesh Networks Supports High Demand, Public Safety and emergency response demand wireless connectivity that supports coverage of large geographic areas, high quality video surveillance and high speed mobility. Wireless Mesh
Networks are great to deliver high throughput, highly reliable wireless connectivity and hence delivers both indoor and outdoor connectivity. Wireless Mesh is an effective, complete solution for covering large areas without sacrificing quality of the wireless network. These Networks are a reliable source of wireless connectivity for a variety of public safety applications, business parks, schools, campus grounds, parking garages, and other large outdoor facilities.

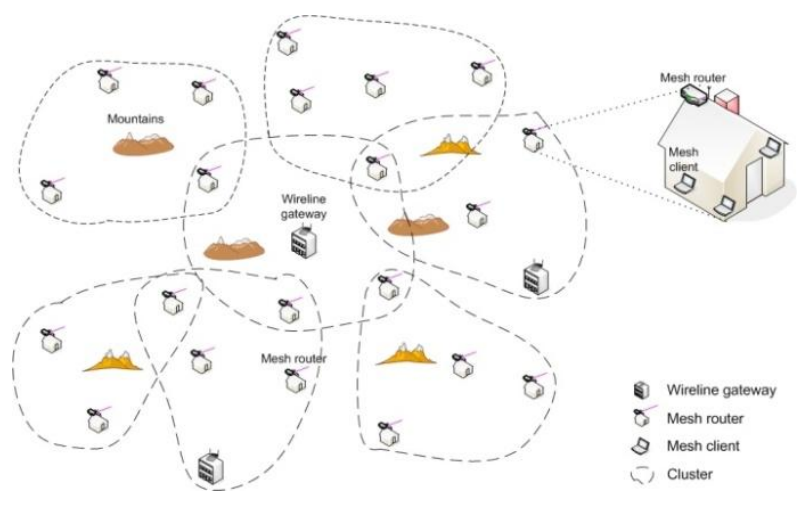

Fig 1: Network Architecture of wireless mesh network

Mesh networks may incorporate either fixed or mobile devices. The solutions are as varied as communication needs, for instance in complex environments such as battlefield surveillance, emergency situations, oil rigs, tunnels, high speed mobile video applications on board public transport or real time racing car telemetry. A significant promising application for wireless mesh networks is VoIP. The Various proactive protocols are Destination-Sequenced Distance Vector Routing Protocol (DSDV) AND Wireless Routing Protocol (WRP) [1]. Reactive protocols do not determine the routes in advance but instead create and maintain routes only on demand .Hence they are in general more scalable in nature [7]. Examples of such protocols are DSR and AODV. Hybrid protocol is a basic routing protocol that is based on AODV and tree-based routing. It supports two types of path selection protocols. It uses peer link management protocol that uses me

\section{LITERATURE REVIEW}

Govindaraj. E. et al. (2012) [7] have proposed a QoS aware robust multipath routing algorithm for wireless mesh networks. The aim of the protocol is to provide a QoS constrained route from source to the destination. So a multiple disjoint paths for a source destination pair. Hence for initial time interval, probe packets are sent along all paths simultaneously from the source to the destination. On receiving the probe packets, cumulative transmission energy, average delay and bandwidth are estimated for each path and 
a feedback report is sent to the destination. Therefore from the multiple paths, the robust best path is selected based on the feedback report from the destination. It also detects the changes in the path quality that hinders QoS requirements of the flows and reroutes the flow through alternative robust routes. Ghosh Saurav et al. (2011) [8] have studied wireless mesh networks as an effective means to provide broadband internet. Connectivity, reliability and throughput being the major QoS concern areas in WMNs. WMNs use orthogonal communication channels and multiple radios to increase throughput, reduce interference and provide path redundancy, connectivity and reliability. S.Kannan et al. (2011) [9] have discussed that Mobile Ad-hoc Networks (MANET) are selforganizing and self-configuring multi-hop wireless networks. Mainly due to the mobility of the nodes, the structure of the network changes dynamically. In mobile ad-hoc networks, a routing procedure is always needed to find a path so as to forward the packets appropriately between the source and the destination. Route changes and temporary link failures occur frequently in a MANET. Assuming that all packet losses are due to congestion, in such an environment TCP performs poorly. So a new mechanism TASR, TCP-aware source routing is proposed that can improve TCP performance in wireless Ad-hoc networks. So to reduce out-of-ordered packets, consecutive timeouts and retransmissions in TCP, TASR adds a hold state to an existing routing protocol. S.Kannan et al. (2010) [10] have studied Mobile Ad-hoc Network routing protocol. The main objective of the protocol is to overcome the hurdles created by the dynamically changing topology and hence create an efficient and correct communication path between any two nodes keeping in view minimum routing overhead and bandwidth consumption. Since an ad-hoc environment offers various challenges that do not exists in fixed networks, the design problem so the routing protocol is not so simple. So a number of routing protocols have been proposed for this purpose. The deep study and the comparison of the performance of the protocols have been done. Various protocols are Destination Sequenced Distance Vector, Ad-Hoc Demand Distance Vector, Dynamic Source Routing, etc. Ishita Bhakta et al. (2010) [11] have studied Wireless mesh networks (WMNs) that consists of mesh clients that can be either stationary or mobile, static mesh routers through which the clients communicate with each other and with the other networks or Internet. Wireless mesh Networks have made their presence felt with a promise of supporting variegated traffic ranging from real-time to best effort while providing coverage to large metropolitan areas. Multi-hop communication, the existence of inherently error prone wireless channel, the static nature of mesh nodes, the availability of sufficient bandwidth resources and the need to transport multimedia traffic with different QoS budgets necessitate the design of QoS-aware routing metrics to improve the performance of routing algorithms. The detailed study of the motivations for new and quality-aware routing metrics has been done and provides with a detailed comparative analysis supported by NS-2simulations of some routing metrics that attempt to address these goals. S.Kannan et al. (2010) [13] have studied and compared the performance of various mobile ad-hoc network routing protocols like AODV, DSI and DSDV. Meeting the challenges of the changing topology, the routing protocol aims at providing a correct and efficient communication path between any two existing nodes of the network hence minimizing bandwidth consumption and routing overhead. S.Karthik et al. (2010) [15] have discussed mobile ad-hoc network as a network that does not require any infrastructure or central administration; hence they are suitable only for providing temporary communication links. The main problem area in computer network is to design the network in such a way that it can efficiently cope up with the speed issue that is mainly required today. In a network the data packets have to be routed to the destination with minimum loss and delay and maximum packet delivery ratio, so there must exist an efficient robust and adaptive routing algorithm that satisfies all the quality of service requirements. Inspired by the behavior of biological ants, Multi Agent Ant Based Routing Algorithm is designed from the ACO framework that consists of both proactive and reactive components. This technique increases packet delivery ratio, node connectivity and decreases average end to end delay. As node connectivity increases, the packet loss is reduced. Ronghui Hou eta al. (2009) [17] has described hopby-hop bandwidth guaranteed routing protocol in IEEE 802.11-based wireless mesh networks. Bandwidth is neither concave nor additive in wireless networks due to the interference among links. Bandwidth needs to be isotonic, the necessary and sufficient property for consistent hop-by-hop routing which is unfortunately not available. So to solve the problem an isotonic parameter is introduced that captures the available bandwidth metric so that packets can traverse the maximum and width path consistently according to the routing tables constructed in the nodes along the path. Chi Harold Liu et al. (2009) [18] have studied Cross-layer design for quality of service (QoS) in wireless mesh networks that are expected to support various types of applications with different and multiple QoS and grade-of-service (GoS) requirements. In order to achieve the same various technologies have been exploited and algorithm needs to be designed. Since most of the existing works on cross-layer design focus on the interaction of up to two layers. While the GoS concept is overlooked in wireless mesh networks, so a unified framework is proposed that exploits both the physical channel properties and multi-user diversity gain of WMNs and by performing intelligent route selection and connection admission control provides both QoS and GoS to a variety of applications.

\section{PROBLEM DEFINATION}

1. Use of WMNs: The various advantages of the mesh network such as self-organising, reliable, robustness, low transmission power etc. have made WMNs to be used in following areas as: Broadband home Networks, Cash Strapped ISPs, Enterprise Networking, Building automation etc. Hence finding its use in various areas.

2. QoS in WMNs: Interference and Noise are the fundamental issues that need to be handled by the Wireless networks due to their broadcasting nature. The QoS of WMNs is more complex than the wired networks due to certain reasons like node mobility, limited battery power, lack of central coordination authority etc. So Qos is still an open challenge in case of WMNs.

3. Multipath Routing Protocol: In the case, the probe packets are being sent from the source to the destination on multiple paths and for each path cumulative transmission energy, bandwidth and average delay is calculated. A feedback report is prepared and evaluated keenly, selecting the most robust path for data transmission.

This research work focuses on good throughput, channel utilization along with reduced energy consumption and delay, so as to maintain QoS in WMNs. 


\section{PROPOSED ALGORITHM}

The proposed algorithm will function in following stages i.e. Cluster formation (Selection of cluster head), compression of data and intra-cluster aggregation(grouping of nodes for transmission of packets to cluster heads for aggregation).

Step 1. Initialization of WMNs: Initially nodes of WMNs ill be deployed in given $\mathrm{x}$ and diameters along with their respective feature i.e. characteristics of the WMNs. As the proposed scenario is based upon the cluster head strategy therefore cluster heads will also be deployed optimistically to cover maximum number of mesh nodes in an optimistic manner. Hence the deployment is done for the nodes in wireless mesh networks in order to send data from the source to the destination in order to get effective data transmission.

Step 2. Initiate Sender: Depends upon the number of mesh service subscribers (MSS), senders will be initiated in this step. However sender node initialization is done on the random variables concept. Where each mesh node has same probability to become sender.

Step 3. Member Mesh Node to Cluster Based Communication: First of all sender node will evaluate the distance between sender mesh node and all the available cluster head using Euclidian distance shown in equation in 1.

distance $=\sqrt{\left(\left(M_{x}-C H_{x}\right)^{2}+\left(M_{y}-C H_{y}\right)^{2}\right)}(1)$

Where $M_{x}$ is the x-axis position of the mesh member node and $M_{y}$ is the $y$-axis position of the mesh member node and also $\mathrm{CH}$ is the $\mathrm{x}$-axis position of the cluster head and $\mathrm{CH}_{y}$ is the $y$-axis position of the cluster head.

Step 4. Intracluster Data Aggregation on Cluster Head(s): Each Cluster head is responsible for aggregating the packets initiated by the different senders i.e. mesh member nodes. Every cluster head has the responsibility to reduce the redundant information of the mesh nodes.

Step 5. Apply Compression On CH: Now loss less LZW based data compression will come in action to reduce the aggregated packet size also called compressed packet size (CPS).

Step 6. Find Optimized Route for Data Transmission using DSDV Protocol: Now DSDV protocol will come in action to find the optimized route for efficient data transmission to the destination mesh node.

Step 7: Repeat step 2to 4 until the simulation time matures.

Step 8. Evaluation Of Performance Metrics:

Now evaluate the performance metrics based upon the predefined formulas.

For channel utilization:

$$
\text { channel utilization }=1-(\text { hopbyhop cost })+\text { toc }
$$

Where toc is turn around time.

$$
\begin{aligned}
& \text { throughput }=(\text { Aggregated packets } \\
& \text { * channelutilization) } \\
& + \text { (Aggregated packets /CPS) }
\end{aligned}
$$

Where CPS is Compressed Packet Size.

For calculation of the delay:

$$
\begin{array}{r}
\text { delay }=\text { toc }+(\text { sum }(\text { endtoend delay }) \\
\left.+\left(C P S * t_{c t s}\right)\right) / \text { counter }
\end{array}
$$

Where $t_{c t s}$ specifies consumed control to send energy for a given node

\section{PRESENT WORK}

In this simulation, Matlab has been used. The clients and the base station are deployed in $1000 \times 1000 \mathrm{~m}$ area for simulation time of 50 seconds. The parameters and settings are summarized in table1.

Table 1: WMNs characteristics

\begin{tabular}{|l|l|}
\hline Parameter & Value \\
\hline Routing Protocol Node Transmission & $250 \mathrm{~m}$ \\
\hline $\begin{array}{l}\text { Range( Node } \\
\text { range) R }\end{array}$ & $1 \mathrm{Mb}$ \\
\hline Rate & 0.0005 \\
\hline Frame Duration & 2000 \\
\hline Packets & 10 \\
\hline No of Nodes & 1500 bytes \\
\hline Packet Size & 3 \\
\hline Cluster & 0 \\
\hline Aggregated Packets & Packet size $/ 5$ \\
\hline CPS & Scale \\
\hline MSS & $0.0001 \mathrm{~J}$ \\
\hline E_r & $0.001 \mathrm{~J}$ \\
\hline t_ack & $0.001 \mathrm{~J}$ \\
\hline t_rts & $0.0001 \mathrm{~J}$ \\
\hline t_cts & $2,4,6,8$ and 10 \\
\hline Clients & \\
\hline
\end{tabular}

The deployment of the network is done initially according to the existing multipath routing algorithm as in fig 2 and then the cluster based routing algorithm technique is used as given in fig 3 . So the simulation area works as:

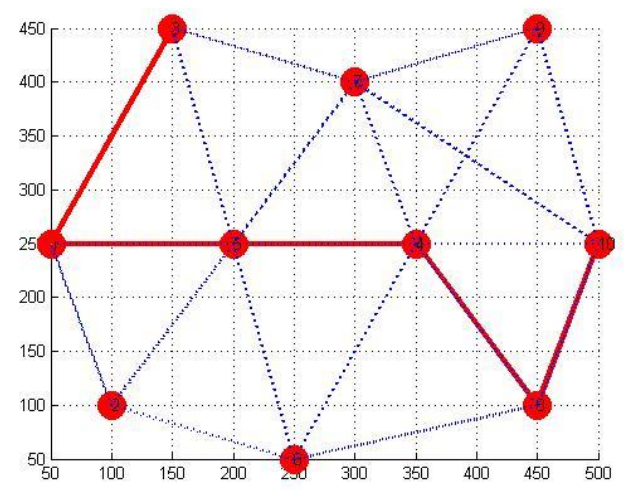

Fig 2: running environment of existing system

For throughput: 


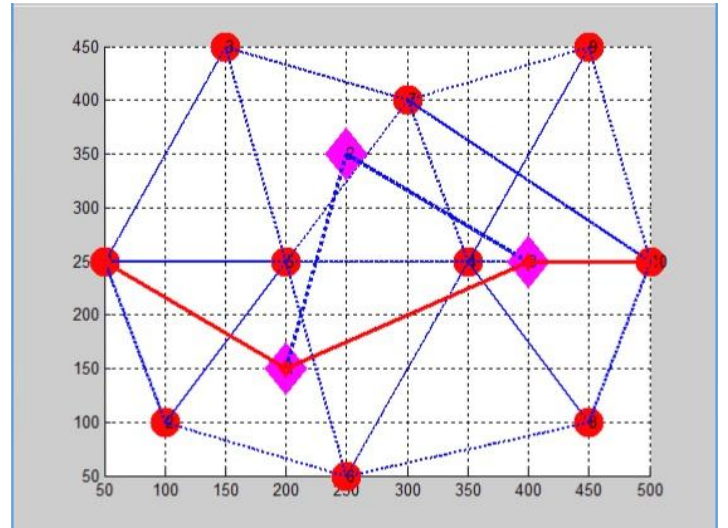

Fig 3: running environment of proposed system

\section{RESULTS}

In this experiment, the no of MSS is varied as 2,4,6,8 and 10 and the results are calculated as:

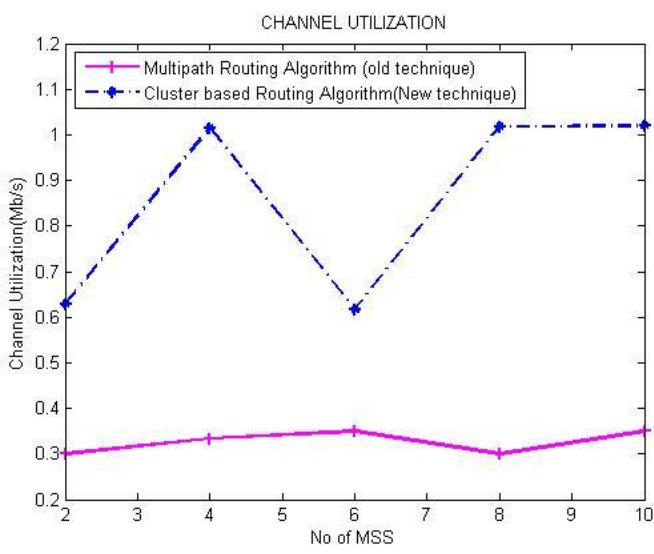

Fig 4: No of MSS Vs channel utilization

Fig 4 shows the key change in the utilization of the channel in the existing and proposed routing algorithm while the data is to be transmitted.

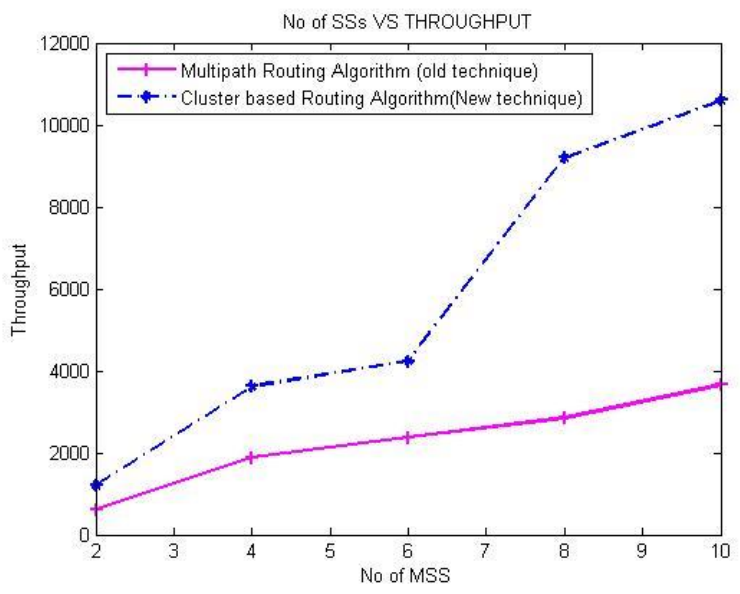

Fig 5: No of MSS Vs throughput

fig 5 represents the throughput calculation of the simulation. It shows the comparison as how much packets are delivered successfully.

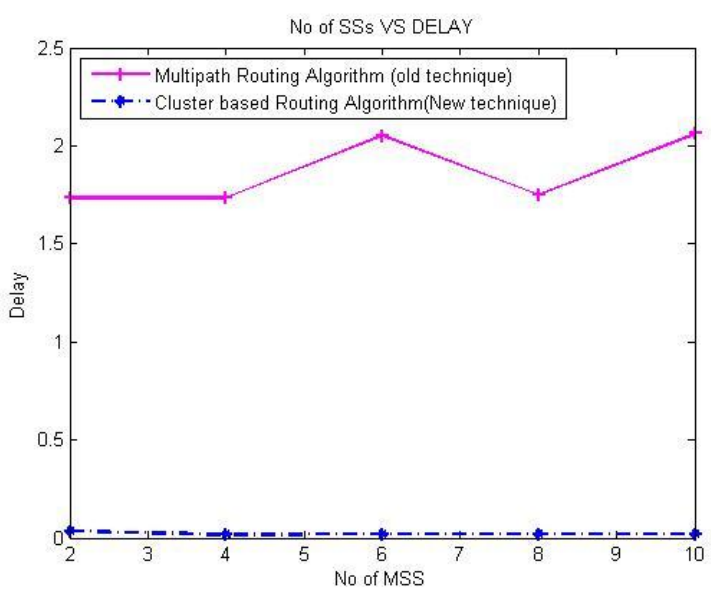

Fig 6: No of MSS Vs delay

The comparison of the end-to-end delay is represented in fig 6 where both the routing algorithms proposed and the existing one are compared. The surviving data packets that reach the destination from the source.

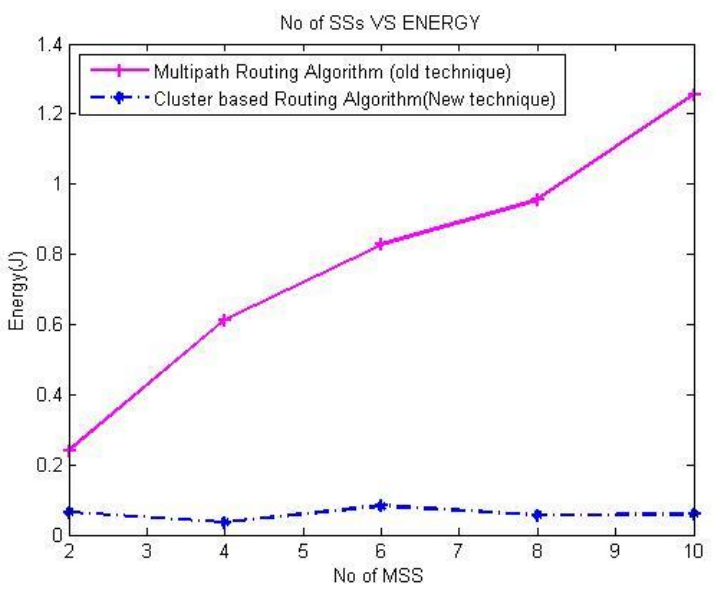

Fig 7: No of MSS Vs energy

The average energy consumption of the nodes for the transmission, receiving and the forwarding of the data packets is shown in fig7. It compares the energy of the system

\section{CONCLUSION AND FUTURE WORK}

This paper has focused on the QoS issue of Wireless mesh networks. QoS teams up with a enormous collection of networking technologies and procedures that guarantee the potential of a network to deliver with predictable consequences. This research work focuses on good throughput, channel utilization along with reduced energy consumption and delay, so as to maintain QoS in WMNs. This paper has proposed a new multi-radio, multi-channel and clustering based wireless mesh protocol to improve the QoS further. This paper has considered the DSDV protocol to locate the secure and optimized path. The proposed technique also utilizes the LZW based lossless data compression and intra cluster data aggregation to enhance the communication between the source and the destination. The use of clustering has the ability to aggregates the multiple packets and locate a single route using the clusters to improves the intra cluster data aggregation. The use of the LZW based loss less data compression has ability to reduce the data packet size so will consume lesser energy thus increase the network QoS. The MATLAB tool has been used to evaluate the effectiveness of the proposed technique. To assess the results and compare 
them with existing results Channel Utilization, Bandwidth use, Average Communication Cost, Throughput, End-to-end delay and Energy metrics of the QoS has been used in this paper. The comparative analysis has shown that the proposed technique outperforms over the available techniques. This paper has not considered the security issue of wireless mesh networks which has found to be very critical issue. So in near future we will evaluate the performance of the proposed technique in case of the various attacks. Also the effect of the attack prevention will also be considered.

\section{REFERENCES}

[1] Gabale, Vijay, Bhaskaran Raman, Partha Dutta, and Shivkumar Kalyanraman. "A classification framework for scheduling algorithms in wireless mesh networks." Communications Surveys \& Tutorials, IEEE 15, no. 1 (2013), pp. 199-222.

[2] Oe, Kiyotaka, Akio Koyama, and Leonard Barolli. "Performance Evaluation of a Multicast Routing Protocol for Wireless Mesh Networks Considering Network Load." In Advanced Information Networking and Applications (AINA),(2013) IEEE 27th International Conference on IEEE(2013), pp. 591-597.

[3] Matam, Rakesh, and Somanath Tripathy. "AFC: An Effective Metric for Reliable Routing in Wireless Mesh Networks." In Trust, Security and Privacy in Computing and Communications (TrustCom), (2013) 12th IEEE International Conference on, IEEE (2013), pp. 73-80.

[4] Naragund, Jayalakshmi G., and R. M. Banakar. "Analysis of HWMP-ETX Routing in Wireless Mesh Networks." In Advanced Computing, Networking and Security (ADCONS), (2013) 2nd International Conference on, IEEE (2013), pp. 208-213.

[5] Mardokhpour, Airin. "Qos-based rate allocation in wireless mesh networks."In International Journal of Computer Science Issues, Vol. 9, Issue 2, No 3 (2012), pp 454-460.

[6] Oe, Kiyotaka, and Akio Koyama. "A Multicast Routing Protocol for Wireless Mesh Networks Considering Network Load." In Network-Based Information Systems (NBiS), (2012) 15th International Conference on, IEEE (2012), pp. 597-602.

[7] E.,Govindaraj. , V. P. Arunachalam, and S. Karthik. "A QoS Aware Robust Multipath Routing Protocol for Wireless Mesh Networks." European Journal of Scientific ResearchVol.78 No.2 (2012), pp.222-231.

[8] Saurav, Ghosh, Das Niva, and Sarkar Tanmoy. "A Cluster Based Multi-Radio Multi-Channel Assignment Approach in Wireless Mesh Networks." In Computer Networks and Information Technologies, Springer Berlin Heidelberg (2011), pp. 487-491.
[9] Kannan, S., S. Karthik, and V. P. Arunachalam. "An Enhanced Packet Retransmission Method for Improving TCP-Aware Source Routing in Mobile Ad-Hoc Network." Asian Journal of Information Technology 10, no. 1 (2011), pp. 20-25.

[10] Kannan, S., S. Karthik, and V. P. Arunachalam. "Performance Analysis and Comparison of Mobile Ad hoc Network Routing Protocols." Research General of Applied Sciences 6, no. 5 (2010), pp. 424-428.

[11] Bhakta, Ishita, Koushik Majumdar, A. Kumar Bhattacharjee, Arik Das, D. Kumar Sanyal, Matangini Chattopadhyay, and Samiran Chattopadhyay. "Incorporating QoS Awareness in Routing Metrics for Wireless Mesh Networks." In Proceedings of the World Congress on Engineering, vol. 1. (2010).

[12] Dolfus, Kirsten, and Torsten Braun. "An evaluation of compression schemes for wireless networks." In Ultra Modern Telecommunications and Control Systems and Workshops (ICUMT), (2010) International Congress on, IEEE (2010), pp. 1183-1188.

[13] Kannan, S., S. Karthik, and V. P. Arunachalam. "An Investigation on Performance Analysis and Comparison of Proactive and Reactive Routing Protocols in Mobile Ad-Hoc Network." International Journal of Soft Computing 5, no. 5 (2010), pp. 194-199.

[14] Marina, Mahesh K., Samir R. Das, and Anand Prabhu Subramanian. "A topology control approach for utilizing multiple channels in multi-radio wireless mesh networks." Computer networks 54, no. 2 (2010), pp. 241256.

[15] Kannan, S., T. Kalaikumaran, S. Karthik, and V. P. Arunachalam. "Ant colony optimization for routing in mobile ad-hoc networks." International Journal of Soft Computing 5, no. 6 (2010), pp. 223-228.

[16] Si, Weisheng, Selvadurai Selvakennedy, and Albert Y. Zomaya. "An overview of channel assignment methods for multi-radio multi-channel wireless mesh networks." Journal of Parallel and Distributed Computing 70, no. 5 (2010), pp. 505-524.

[17] Hou, Ronghui, King-Shan Lui, Hon-Sun Chiu, Kwan L. Yeung, and Fred Baker. "Routing in multi-hop wireless mesh networks with bandwidth guarantees." In Proceedings of the tenth ACM international symposium on Mobile ad hoc networking and computing, ACM (2009), pp. 353-354.

[18] Liu, Chi Harold, Athanasios Gkelias, Yun Hou, and Kin K. Leung. "Cross-layer design for QoS in wireless meshes networks." Wireless personal communications 51, no. 3 (2009), pp. 593-613. 